\title{
On the structure of the Selberg class, VI: non-linear twists
}

\author{
by \\ J. Kaczorowski (Poznań) and A. Perelli (Genova)
}

1. Introduction. In the first paper of the series, [7], we investigated the analytic properties of the linear twists

$$
F(s, \alpha)=\sum_{n=1}^{\infty} \frac{a(n)}{n^{s}} e(-n \alpha), \quad e(x)=e^{2 \pi i x}, \alpha \in \mathbb{R}, \sigma>1,
$$

of functions $F(s)$ of degree 1 in the extended Selberg class $\mathcal{S}^{\sharp}$. Precisely, denoting by $q_{F}$ and $\theta_{F}$ respectively the conductor and the shift of $F(s)$ (see below for definitions) and writing $n_{\alpha}=q_{F} \alpha$, we proved that for $\alpha>0$ the twist $F(s, \alpha)$ has meromorphic continuation to $\sigma>0$, and it has a simple pole at $s=1-i \theta_{F}$ if and only if $n_{\alpha} \in \mathbb{N}$ and $a\left(n_{\alpha}\right) \neq 0$ (see Theorem 7.1 of [7]). In [7] we exploited such analytic properties in order to characterize the functions of degree 1 in $\mathcal{S}^{\sharp}$. In particular, we proved that the only functions of degree 1 in the Selberg class $\mathcal{S}$ are the Riemann zeta function $\zeta(s)$ and the shifted Dirichlet $L$-functions $L(s+i \theta, \chi)$, with $\theta \in \mathbb{R}$ and primitive characters $\chi$.

It turns out that Theorem 7.1 of [7] is a special case of a general result for functions in $\mathcal{S}^{\sharp}$ of any degree $d>0$. To see this, for $d>0, \alpha \in \mathbb{R}$ and $F \in \mathcal{S}^{\sharp}$ with degree $d$ we consider the non-linear twist

$$
F(s, \alpha)=\sum_{n=1}^{\infty} \frac{a(n)}{n^{s}} e\left(-n^{1 / d} \alpha\right), \quad \sigma>1 ;
$$

note that $F(s, \alpha)$ in (1.2) becomes the linear twist in (1.1) when $d=1$. In this paper we first obtain the basic analytic properties of $F(s, \alpha)$ and some uniform bounds on vertical strips, and then we turn to various applications.

We refer to the survey paper [8] and to [7] for the basic notation and properties of the Selberg class $\mathcal{S}$ and the extended class $\mathcal{S}^{\sharp}$. Here we recall that $\mathcal{S}$ denotes the class of Dirichlet series $F(s)$ satisfying the following five axioms: (i) the abscissa of absolute convergence $\sigma_{a}(F)$ satisfies $\sigma_{a}(F) \leq 1$;

2000 Mathematics Subject Classification: Primary 11M41. 
(ii) $(s-1)^{m} F(s)$ is an entire function of finite order for some integer $m \geq 0$; (iii) $F(s)$ satisfies a functional equation of the form

$$
\Phi(s)=\omega \bar{\Phi}(1-s)
$$

where $|\omega|=1, \bar{f}(s)=\overline{f(\bar{s})}$ and

$$
\Phi(s)=Q^{s} \prod_{j=1}^{r} \Gamma\left(\lambda_{j} s+\mu_{j}\right) F(s)=\gamma(s) F(s),
$$

say, with $Q>0, \lambda_{j}>0$ and $\Re \mu_{j} \geq 0$; (iv) the Dirichlet coefficients $a(n)$ of $F(s)$ satisfy the Ramanujan conjecture $a(n) \ll n^{\varepsilon}$ for every $\varepsilon>0$; (v) $\log F(s)$ is a Dirichlet series with coefficients $b(n)$ satisfying $b(n)=0$ unless $n=p^{m}, m \geq 1$, and $b(n) \ll n^{\vartheta}$ for some $\vartheta<1 / 2$. Moreover, $\mathcal{S}^{\sharp}$ denotes the extended Selberg class, consisting of the non-zero functions satisfying only the first three axioms, $m_{F} \geq 0$ denotes the order of pole at $s=1$ of $F \in \mathcal{S}^{\sharp}$ and $\gamma(s)$ is called a $\gamma$-factor. We also recall that the degree, $\xi$-invariant, conductor and root number of $F \in \mathcal{S}^{\sharp}$ are defined by

$$
\begin{gathered}
d_{F}=2 \sum_{j=1}^{r} \lambda_{j}, \quad \xi_{F}=2 \sum_{j=1}^{r}\left(\mu_{j}-\frac{1}{2}\right)=\eta_{F}+i \theta_{F}, \\
q_{F}=(2 \pi)^{d_{F}} Q^{2} \prod_{j=1}^{r} \lambda_{j}^{2 \lambda_{j}}, \quad \omega_{F}^{*}=\omega e^{-i \frac{\pi}{2}\left(\eta_{F}+1\right)}\left(\frac{q_{F}}{(2 \pi)^{d_{F}}}\right)^{i \frac{\theta_{F}}{d_{F}}} \prod_{j=1}^{r} \lambda_{j}^{-2 i \Im \mu_{j}},
\end{gathered}
$$

respectively, and that $\mathcal{S}_{d}\left(\right.$ resp. $\mathcal{S}_{d}^{\sharp}$ ) denotes the subclass of $\mathcal{S}$ (resp. $\mathcal{S}^{\sharp}$ ) consisting of the functions of given degree $d$. Further, we will use $f(x) \asymp g(x)$ to mean $g(x) \ll f(x) \ll g(x)$ and $s=\sigma+i t$, and the value of the constant $c$ below will not necessarily be the same at each occurrence.

For $d>0, F \in \mathcal{S}_{d}^{\sharp}$ and $\alpha>0$ we write

$$
n_{\alpha}=q_{F} d^{-d} \alpha^{d}, \quad a\left(n_{\alpha}\right)= \begin{cases}0 & \text { if } n_{\alpha} \notin \mathbb{N}, \\ a\left(n_{\alpha}\right) & \text { if } n_{\alpha} \in \mathbb{N} .\end{cases}
$$

The basic analytic properties of $F(s, \alpha)$ are given by the following general version of Theorem 7.1 of [7].

Theorem 1. Let $d>0, F \in \mathcal{S}_{d}^{\sharp}$ and $\alpha>0$. Then $F(s, \alpha)$ has meromorphic continuation to $\mathbb{C}$. Moreover, $F(s, \alpha)$ is entire if $a\left(n_{\alpha}\right)=0$, while if $a\left(n_{\alpha}\right) \neq 0$ then $F(s, \alpha)$ has at most simple poles at the points

$$
s_{k}=\frac{d+1}{2 d}-\frac{k}{d}-i \frac{\theta_{F}}{d}, \quad k=0,1, \ldots,
$$

with non-vanishing residue at $s_{0}$.

It is well known that $\mathcal{S}_{d}^{\sharp}=\emptyset$ for $0<d<1$ (see for example Corollary 5 below). Nevertheless, we still consider the case $0<d<1$ since Theorem 1 allows us to obtain a simple new proof of the non-existence of functions 
$F \in \mathcal{S}_{d}^{\sharp}$ with $d$ in that range (see again Corollary 5 below). Moreover, we remark that it is in principle possible to check the vanishing or non-vanishing of the residue at each point $s_{k}$, but there are non-trivial complications in details (see Lemmas 2.4, 2.5 and the proof of Theorem 1 below).

With applications in mind, we now turn to certain uniform bounds for $F(s, \alpha)$. To this end we call a family $\mathcal{F}$ of $L$-functions in $\mathcal{S}^{\sharp}$ admissible if every $F \in \mathcal{F}$ has $d_{F} \ll 1, m_{F} \ll 1$, a $\gamma$-factor with

$$
Q \gg 1, \quad \lambda_{j} \gg 1, \quad \mu_{j} \ll 1
$$

and, moreover, satisfies

$$
\sum_{n \leq x}|a(n)| \ll x^{1+\varepsilon}
$$

where the implied constants in the $\ll$-symbols depend on the family $\mathcal{F}$. Note that each $F \in \mathcal{F}$ has $q_{F} \asymp_{\mathcal{F}} Q^{2}$ and $d_{F}>0$ (and hence $d_{F} \geq 1$ by, e.g., Corollary 5 below). We remark that, conjecturally, $d_{F} \ll 1$ implies $m_{F} \ll 1$, and every $F \in \mathcal{F}$ has a $\gamma$-factor with $Q \gg 1$ and $\lambda_{j} \gg 1$. Moreover, if all $F \in \mathcal{F} \subset \mathcal{S}$ have polynomial Euler product with uniformly bounded degree, then (1.3) is automatically satisfied and $m_{F} \ll 1$. Examples of admissible families are a single $F \in \mathcal{S}^{\sharp}$ with $d_{F} \geq 1$, the set of Dirichlet $L$-functions associated with primitive characters, the set of normalized $L$-functions associated with modular forms of bounded weight, and the set of Dedekind zeta functions of number fields with bounded degree. Note, however, that for a fixed primitive Dirichlet character $\chi$ the set $\{L(s+i k, \chi)\}_{k \in \mathbb{Z}}$ is not an admissible family, nor is the set of all Dedekind zeta functions or even the set of integer multiples of $\zeta(s)$.

Let $\mathcal{F}$ be an admissible family and $\Delta \geq 2$. We remark here that for $|s-1| \geq 1 / 4$ the functional equation and the Phragmén-Lindelöf theorem imply the bound

$$
F(s) \ll \mathcal{F}, \Delta q_{F}^{c-\sigma}(|t|+2)^{c \Delta}
$$

uniformly for $F \in \mathcal{F}$ and $-\Delta \leq \sigma \leq 2$, where $c=c(\mathcal{F})$.

Given $F \in \mathcal{S}^{\sharp}$ we denote by $\langle x\rangle$ the distance of $x$ from the nearest integer $n$ such that $a(n) \neq 0$. Moreover, for $\alpha>0$ we write

$$
\delta(\alpha)= \begin{cases}\alpha & \text { if } a\left(n_{\alpha}\right) \neq 0 \\ \alpha /\left\langle n_{\alpha}\right\rangle & \text { if } a\left(n_{\alpha}\right)=0 .\end{cases}
$$

The required uniform bounds on vertical strips are given by

TheOREM 2. Let $\mathcal{F}$ be an admissible family, $\alpha>0, \Delta \geq 2$ and $s_{k}$ be as in Theorem $1(k=0,1, \ldots)$. Then for $\left|s-s_{k}\right| \geq 1 / 4 d_{F}$ we have

$$
F(s, \alpha) \ll \mathcal{F}, \Delta q_{F}^{c-\sigma}(|t|+2)^{c \Delta} \delta(\alpha)^{c \Delta}
$$


uniformly for $F \in \mathcal{F},-\Delta \leq \sigma \leq 2$ and $n_{\alpha} \gg_{\mathcal{F}} 1$, where $c=c(\mathcal{F})$. Moreover

$$
\operatorname{res}_{s=s_{k}} F(s, \alpha)=\omega_{F}^{*} \overline{a\left(n_{\alpha}\right)} q_{F}^{1 / 2-s_{k}} n_{\alpha}^{s_{k}-1} c_{k}(F), \quad k=0,1, \ldots,
$$

where $c_{k}(F)=O_{\mathcal{F}, k}(1)$ uniformly for $F \in \mathcal{F}$ and $c_{0}(F) \neq 0$.

We remark here that the main goal of this paper is the description of the analytic structure of $F(s, \alpha)$ presented in Theorem 1, and that we do not attempt to get sharp uniform bounds for $F(s, \alpha)$. Sharper bounds than (1.5), especially in the $\alpha$-aspect, can be obtained by refining the arguments in Sections 2 and 3 below.

Our first application deals with the following non-linear exponential sum associated with the coefficients of $F \in \mathcal{S}_{d}^{\sharp}$. Let $\phi(u)$ be a smooth function on $(0, \infty)$ with compact support, $\widetilde{\phi}(s)$ its Mellin transform, $\alpha>0, x>1$ and

$$
S_{F}(x)=S_{F}(x, \alpha ; \phi)=\sum_{n=1}^{\infty} a(n) e\left(-n^{1 / d} \alpha\right) \phi\left(\frac{n}{x}\right) .
$$

A standard application of Theorem 2 gives

Corollary 1. Let $\mathcal{F}$ be an admissible family and $\alpha>0$. Then for every $A>0$ we have

$$
\begin{aligned}
S_{F}(x)= & \omega_{F}^{*} \frac{\overline{a\left(n_{\alpha}\right)}}{n_{\alpha}} q_{F}^{1 / 2} \sum_{0 \leq k \leq d_{F} A+\left(d_{F}+1\right) / 2} c_{k}(F) \widetilde{\phi}\left(s_{k}\right)\left(\frac{x n_{\alpha}}{q_{F}}\right)^{s_{k}} \\
& +O_{\mathcal{F}, \phi, A}\left(q_{F}^{c+A} \delta(\alpha)^{c A} x^{-A}\right)
\end{aligned}
$$

uniformly for $F \in \mathcal{F}$ and $n_{\alpha} \gg_{\mathcal{F}} 1$, where $c=c(\mathcal{F})$.

Corollary 1 should be compared with the results on the corresponding non-linear exponential sum

$$
S_{q}(x)=\sum_{n=1}^{\infty} a_{n} e(-2 \sqrt{n q}) \phi\left(\frac{n}{x}\right), \quad q>0,
$$

obtained in Appendix C of Iwaniec-Luo-Sarnak [6], and in particular with the asymptotic formula (C.17) of [6] where the coefficients $a_{n}$ come from a degree $2 L$-function. Note that the parameter $q$ in (1.6) corresponds to our parameter $\alpha$. Apart from the degree of uniformity in $\alpha$ (but see the remark after Theorem 2), Corollary 1 improves and extends such results. Note also that our approach is different from [6], and that in view of the pole of $F(s, \alpha)$ at $s=s_{0}$, Theorem 1 gives some further support to the conditional asymptotic formula (C.33) of [6], dealing with a non-linear exponential sum similar to (1.6), but over primes.

The next application deals with an $\Omega$-result for

for any $F \in \mathcal{S}_{d}^{\sharp}$ with $d \geq 1$.

$$
A_{F}(x)=\sum_{n \leq x} a(n)
$$


Corollary 2. Let $F \in \mathcal{S}_{d}^{\sharp}$ with $d \geq 1$. Then for every polynomial $P \in \mathbb{C}[x]$ we have

$$
A_{F}(x)=x P(\log x)+\Omega\left(x^{(d-1) / 2 d}\right) .
$$

Clearly, the interesting case in Corollary 2 is when $P(\log x)$ is chosen as in (4.2) below. We remark that Corollary 2 can possibly be proved in a classical way by means of Voronoï-type expansions, although we could not trace this result (in the full generality of the class $\mathcal{S}^{\sharp}$ ) in ChandrasekharanNarasimhan [3] and related papers. Moreover, the proof of Corollary 2 clearly shows that the exponent in the $\Omega$-estimate is caused by the pole of $F(s, \alpha)$ (with a suitable choice of $\alpha$ ) at $s=s_{0}$.

Let $\sigma_{c}(F)$ denote the abscissa of convergence of $F \in \mathcal{S}_{d}^{\sharp}$. We have

Corollary 3. Let $F \in \mathcal{S}_{d}^{\sharp}$ with $d \geq 1$. Then

$$
\sigma_{c}(F) \geq \frac{d-1}{2 d} .
$$

In the same way as Corollary 3 we can prove the following generalization. For $F \in \mathcal{S}_{d}^{\sharp}$ with $d \geq 1$ and any polynomial $Q \in \mathbb{C}[x]$, the abscissa of convergence $\sigma_{c}(F, Q)$ of the series

$$
\sum_{n=1}^{\infty} \frac{a(n)-Q(\log n)}{n^{s}}
$$

satisfies $\sigma_{c}(F, Q) \geq(d-1) / 2 d$.

Corollary 3, our result that $\mathcal{S}_{d}^{\sharp}=\emptyset$ for $1<d<5 / 3$ (see [9]) and the description of the functions of degree 1 in $\mathcal{S}$ (see above and Theorem 3 of [7]) allow us to obtain the following characterizations of the Riemann zeta function and of the Dirichlet $L$-functions. Our proof is quite indirect, and we wonder if there exists a simpler and direct proof.

Corollary 4. Let $F \in \mathcal{S}_{d}$ with $d \geq 1$. If the series

$$
\sum_{n=1}^{\infty} \frac{a(n)-1}{n^{s}} \quad\left(\text { resp. } \sum_{n=1}^{\infty} \frac{a(n)}{n^{s}}\right)
$$

converges for $\sigma>1 / 5-\delta$ with some $\delta>0$, then $F(s)=\zeta(s)($ resp. $F(s)=$ $L(s+i \theta, \chi)$ with some $\theta \in \mathbb{R}$ and a primitive Dirichlet character $\chi)$.

Our last application is another proof of the well known result asserting that there are no functions in $\mathcal{S}_{d}^{\sharp}$ with $0<d<1$; see Richert [11], Bochner [1], Conrey-Ghosh [4] and Molteni [10] for several proofs.

Corollary 5. $\mathcal{S}_{d}^{\sharp}=\emptyset$ for $0<d<1$.

In fact, suppose that there exists $F \in \mathcal{S}_{d}^{\sharp}$ with $0<d<1$ and let $a(m) \neq 0$ for some integer $m$. Choosing $\alpha$ such that $n_{\alpha}=m$, from Theorem 1 we 
deduce that $F(s, \alpha)$ has a pole at $s_{0}=(d+1) / 2 d-i \theta_{F} / d$ with $\Re s_{0}>1$, a contradiction since $F(s, \alpha)$ is absolutely convergent for $\sigma>1$. We remark that the nature of this proof is different from those quoted above. Indeed, our proof depends on the polar structure of the associated non-linear twist $F(s, \alpha)$, while all the above quoted proofs depend on the behavior of the coefficients $a(n)$ of $F(s)$.

Acknowledgments. This research was partially supported by Istituto Nazionale di Alta Matematica, by a MURST grant and by KBN grant 1 PO3A 00826.

2. Hypergeometric functions. Let $\boldsymbol{\lambda}=\left(\lambda_{1}, \ldots, \lambda_{r}\right)$ with $\lambda_{j}>0$, $\boldsymbol{\mu}=\left(\mu_{1}, \ldots, \mu_{r}\right)$ with $\mu_{j} \in \mathbb{C}, d=2 \sum_{j=1}^{r} \lambda_{j}$ and $K \geq 1$ be an integer. We consider the incomplete Fox hypergeometric function

$$
H_{K}(z, s)=H_{K}(z, s ; \boldsymbol{\lambda}, \boldsymbol{\mu})=\frac{1}{2 \pi i} \int_{(-K-1 / 2)} h(w, s) z^{w} d w,
$$

where $h(w, s)=h(w, s ; \boldsymbol{\lambda}, \boldsymbol{\mu})=h^{*}(w, s) \Gamma(w)$,

$$
h^{*}(w, s)=h^{*}(w, s ; \boldsymbol{\lambda}, \boldsymbol{\mu})=\prod_{j=1}^{r} \frac{\Gamma\left(\lambda_{j}(1-s)+\bar{\mu}_{j}-\frac{\lambda_{j}}{d} w\right)}{\Gamma\left(\lambda_{j} s+\mu_{j}+\frac{\lambda_{j}}{d} w\right)}
$$

and $z^{w}=e^{w l(z)}, l(z)$ denoting the branch of $\log z$ on $\mathbb{C} \backslash(-\infty, 0]$ satisfying $|\Im l(z)|<\pi$. In Sections 4-6 of [7] we studied the analytic properties of the functions $H_{K}(z, s)$ for $0<\sigma<2$, in the case $d=1$ and $\Re \mu_{j} \geq 0$. In order to prove Theorems 1 and 2 we need to extend the study of $H_{K}(z, s)$ to the half-plane $\sigma<2$ and to obtain uniform bounds on vertical strips, in the general case $d>0$ and $\Re \mu_{j} \geq 0$.

We first remark that a simple transformation shows that the general case $d>0$ can be reduced to the case $d=1$. In fact, writing

$$
\begin{array}{lll}
\widetilde{s}=d s-\frac{d-1}{2}, & \widetilde{\lambda}_{j}=\frac{\lambda_{j}}{d}, & \widetilde{\mu}_{j}=\mu_{j}+\frac{\lambda_{j}}{2}\left(1-\frac{1}{d}\right), \\
\widetilde{d}=2 \sum_{j=1}^{r} \widetilde{\lambda}_{j}=1, & \widetilde{\boldsymbol{\lambda}}=\left(\widetilde{\lambda}_{1}, \ldots, \widetilde{\lambda}_{r}\right), & \widetilde{\boldsymbol{\mu}}=\left(\widetilde{\mu}_{1}, \ldots, \widetilde{\mu}_{r}\right),
\end{array}
$$

we have

$$
H_{K}(z, s ; \boldsymbol{\lambda}, \boldsymbol{\mu})=H_{K}(z, \widetilde{s} ; \widetilde{\boldsymbol{\lambda}}, \widetilde{\boldsymbol{\mu}}),
$$

thanks to the identities

$$
\begin{gathered}
\widetilde{\lambda}_{j}(1-\widetilde{s})+\overline{\widetilde{\mu}}_{j}-\frac{\widetilde{\lambda}_{j}}{\widetilde{d}} w=\lambda_{j}(1-s)+\bar{\mu}_{j}-\frac{\lambda_{j}}{d} w \\
\widetilde{\lambda}_{j} \widetilde{s}+\widetilde{\mu}_{j}+\frac{\widetilde{\lambda}_{j}}{\widetilde{d}} w=\lambda_{j} s+\mu_{j}+\frac{\lambda_{j}}{d} w .
\end{gathered}
$$


Note that $\widetilde{d}=1$ and $\widetilde{\lambda}_{j}>0$, while the $\Re \widetilde{\mu}_{j}$ 's are not necessarily non-negative when $0<d<1$, even if $\Re \mu_{j} \geq 0$. However, the non-negativity of the $\boldsymbol{\mu}$-data is not important in our study of the hypergeometric functions, and hence (2.3) shows that we may restrict ourselves to the study of $H_{K}(z, s)$ in the case $d=1$ and $\mu_{j} \in \mathbb{C}$. Therefore, unless explicitly remarked, in the rest of this section we will assume that $d=1$ but we will not assume the condition $\Re \mu_{j} \geq 0$ for $j=1, \ldots, r$.

Since in view of transformation (2.3) we need to obtain the analytic properties of $H_{K}(z, s)$ in the half-plane $\sigma<R$ with a suitable constant $R \geq 2$, we require that the poles of $h(w, s)$ have positive distance from the line $\Re w=-K-1 / 2$ for every $s$ with $\sigma \leq R$. Clearly, the choice

$$
K=\left[\max _{j=1, \ldots, r} \frac{1+2\left|\mu_{j}\right|}{2 \lambda_{j}}+2 \sum_{j=1}^{r}\left(\left|\mu_{j}\right|+\frac{1}{2}\right)+R\right]+1
$$

ensures this (note that the full force of this choice will be used later on, especially in Lemma 2.4 below). Moreover, with abuse of notation, in analogy with the definition of an admissible family $\mathcal{F} \subset \mathcal{S}^{\sharp}$ we say that a family $\mathcal{F}$ of data $(\boldsymbol{\lambda}, \boldsymbol{\mu})$ is admissible if $d=1, \lambda_{j} \gg 1$ and $\mu_{j} \ll 1$, where the implied constants depend on $\mathcal{F}$. Clearly, by (2.2) every admissible family of $L$-functions in $\mathcal{S}^{\sharp}$ induces an admissible family of data $(\widetilde{\boldsymbol{\lambda}}, \widetilde{\boldsymbol{\mu}})$.

For clarity of exposition, we recall here the basic result on the analytic continuation of $H_{K}(z, s)$ obtained in [7]. Let

$$
\beta=\prod_{j=1}^{r} \lambda_{j}^{-2 \lambda_{j}}
$$

be the $\beta$-parameter of $H_{K}(z, s)$ and

$$
\begin{aligned}
A & =\{z \in \mathbb{C}: \Re z>0\}, \\
B_{\beta} & =\{z \in \mathbb{C}:|z|<1 / \beta\} \backslash(-1 / \beta, 0], \\
C_{\beta} & =\{z \in \mathbb{C}:|z|>1 / \beta\}, \\
D_{\beta} & =A \cup B_{\beta} \cup C_{\beta} .
\end{aligned}
$$

Lemma 2.1. Let $d=1, R \geq 2$ and $K$ be as in (2.4). Then integral (2.1) is absolutely and uniformly convergent on compact sets of $A \times\{\sigma<R\}$ and $H_{K}(z, s)$ has holomorphic continuation to $D_{\beta} \times\{\sigma<R\}$ as a single-valued function.

Proof. This is essentially Theorem 4.1 of [7], with the domain $\Omega$ replaced by the half-plane $\{\sigma<R\}$ and $\mu_{j} \in \mathbb{C}$; the proof is exactly the same.

In particular, from Lemma 2.1 we see that the function $H_{K}(-i y, s)$ is holomorphic for $\sigma<R$, provided $y>0$ with $y \neq 1 / \beta$. Since in our applications to the study of the non-linear twist $F(s, \alpha)$ we need to have control 
on $H_{K}(-i y, s)$ for every $y>0$, for $\sigma<R$ we write

$$
H_{K}(-i / \beta, s)=\lim _{z \rightarrow-i / \beta} H_{K}(z, s),
$$

where the limit is taken along a path where $\Re z>0$. In Sections 5 and 6 of $[7]$ we proved, in the restricted range $0<\sigma<2$, that the limit exists and does not depend on the path. Moreover, we studied the function $H_{K}(-i / \beta, s)$ in the strip $0<\sigma<2$, and in particular its polar structure (see Theorems 5.1 and 6.1 of [7]). In order to extend such results and to get uniform bounds we need several lemmas. Although Lemma 2.1 already shows that $H_{K}(-i y, s)$ is holomorphic for $\sigma<R$ if $y>0$ with $y \neq 1 / \beta$, for completeness we include a sketch of proof.

For $K$ as in (2.4) and $z=-i y$ we write

$$
\begin{aligned}
I(V ; z, s) & =\frac{1}{2 \pi i} \int_{-K-1 / 2-i V}^{-K-1 / 2+i V} h(w, s) z^{w} d w \\
J^{ \pm}(V ; z, s) & =\frac{1}{2 \pi i} \int_{-\infty \pm i V}^{-K-1 / 2 \pm i V} h(w, s) z^{w} d w \\
\widetilde{J}^{ \pm}(V ; z, s) & =\frac{1}{2 \pi i} \int_{-K-1 / 2 \pm i V}^{\infty \pm i V} h(w, s) z^{w} d w
\end{aligned}
$$

and prove

LEMMA 2.2. Let $\mathcal{F}$ be an admissible family of data, $L \geq 2, y>0$, $R=R(\mathcal{F}) \geq 2, K$ be as in (2.4) and $V>0$ be sufficiently large. Then for $(\boldsymbol{\lambda}, \boldsymbol{\mu}) \in \mathcal{F}$ the integral $I(V ;-i y, s)$ is holomorphic for $\sigma \leq R$, while $J^{ \pm}(V ;-i y, s)$ (with $\left.y>1 / \beta\right)$ and $\widetilde{J}^{ \pm}(V ;-i y, s)$ (with $\left.0<y<1 / \beta\right)$ are holomorphic for $-L \leq \sigma \leq R$ and $t \in \mathbb{R}$ satisfying $V \geq c(|t|+L)^{3}$ with a suitable $c=c(\mathcal{F})$. Moreover, for $V \geq c(|t|+L)^{3}$ we have

$$
\begin{aligned}
I(V ;-i y, s) & \ll \mathcal{F}, L V^{c L} y^{-K-1 / 2}, \\
J^{ \pm}(V ;-i y, s) & \ll \mathcal{F}, L \frac{V^{c L} y^{-K-1 / 2}}{\log \beta y}
\end{aligned}
$$

if $y>1 / \beta$ and $V \geq c \log ^{-6} \beta y$, and

$$
\widetilde{J}^{ \pm}(V ;-i y, s) \ll \mathcal{F}, L \frac{V^{c L} y^{-K-1 / 2}}{|\log \beta y|}
$$

if $0<y<1 / \beta$, uniformly for $(\boldsymbol{\lambda}, \boldsymbol{\mu}) \in \mathcal{F}$ and $-L \leq \sigma \leq R$, where $c=c(\mathcal{F})$.

Proof. We first remark that the constants implied in the «-symbols below may depend on $\mathcal{F}$ and $L$. Moreover, in view of (2.4) and condition $V \geq c(|t|+L)^{3}$, the paths in the integrals under consideration have positive 
distance from the poles of $h(w, s)$. Further, we only prove the bounds for such integrals, since their holomorphy follows by the same argument.

We start with the $I$-integral. Writing $w=-K-1 / 2+i v$ with $-V \leq$ $v \leq V$, by the identity

we get

$$
\Gamma(z) \Gamma(1-z)=\frac{\pi}{\sin \pi z}
$$

$$
\begin{aligned}
h^{*}(w, s) \ll & \prod_{j=1}^{r} \Gamma\left(\lambda_{j}(1-s)+\bar{\mu}_{j}-\lambda_{j} w\right) \Gamma\left(1-\lambda_{j} s-\mu_{j}-\lambda_{j} w\right) \\
& \times \sin \pi\left(\lambda_{j} s+\mu_{j}+\lambda_{j} w\right) \\
= & \prod_{j=1}^{r} \Gamma\left(a_{j}-i \lambda_{j}(t+v)\right) \Gamma\left(b_{j}-i \lambda_{j}(t+v)\right) \sin \pi\left(c_{j}+i \lambda_{j}(t+v)\right),
\end{aligned}
$$

where $a_{j}=\lambda_{j}(K+3 / 2-\sigma)+\bar{\mu}_{j}, b_{j}=\lambda_{j}(K+1 / 2-\sigma)+1-\mu_{j}, c_{j}=$ $\lambda_{j}(\sigma-K-1 / 2)+\mu_{j}$. Note that for $\sigma \leq R$ we have $\Re a_{j}, \Re b_{j}>0$ by $(2.4)$. Clearly

$$
\sin \pi\left(c_{j}+i \lambda_{j}(t+v)\right) \ll e^{\pi \lambda_{j}|t+v|}
$$

and by Stirling's formula

$$
\Gamma\left(a_{j}-i \lambda_{j}(t+v)\right) \Gamma\left(b_{j}-i \lambda_{j}(t+v)\right) \ll e^{-\pi \lambda_{j}|t+v|}(1+|t+v|)^{c L}
$$

uniformly for $(\boldsymbol{\lambda}, \boldsymbol{\mu}) \in \mathcal{F}$ and $-L \leq \sigma \leq R$, where $c=c(\mathcal{F})$. Therefore, recalling that the value of $c$ is not necessarily the same at each occurrence, we have

$$
h^{*}(w, s) \ll(1+|t+v|)^{c L}
$$

and hence, again by Stirling's formula,

$$
I(V ;-i y, s) \ll \int_{-V}^{V}(1+|t+v|)^{c L}(1+|v|)^{c} e^{-\frac{\pi}{2}|v|} y^{-K-1 / 2} e^{\frac{\pi}{2}|v|} d v \ll V^{c L} y^{-K-1 / 2}
$$

provided $V \geq c(|t|+L)^{3}$, say, as required.

Now we turn to the $J^{ \pm}$-integrals; we consider only the $J^{+}$-integral since both the treatment of the $J^{-}$-integral and the resulting bound are the same. Also, by the same reason we assume that $t \geq 0$. Writing $w=u+i V$ with $u \leq-K-1 / 2$, arguing as for the $I$-integral and recalling that $d=1$ we have

$$
\begin{aligned}
h^{*}(w, s) \ll e^{\frac{\pi}{2}(t+V)} \prod_{j=1}^{r} \mid \Gamma\left(\lambda_{j}(1-s)\right. & \left.+\bar{\mu}_{j}+\lambda_{j}(|u|-i V)\right) \\
& \times \Gamma\left(1-\lambda_{j} s-\mu_{j}+\lambda_{j}(|u|-i V)\right) \mid .
\end{aligned}
$$


Here we use the following form of Stirling's formula:

$$
\log \Gamma(z+a)=(z+a-1 / 2) \log z-z+O(1)
$$

for $|z|$ sufficiently large, uniformly for $|a| \leq|z|^{1 / 3}$ and $|\arg z| \leq \pi-\varepsilon$, for any fixed $\varepsilon>0$. Since (2.6) can be proved starting with the classical Stirling formula and performing standard manipulations, we omit the proof. Taking $z=\lambda_{j}(|u|-i V)$ and $a=\lambda_{j}(1-s)+\bar{\mu}_{j}$ or $a=1-\lambda_{j} s-\mu_{j}$ we have $|\arg z| \leq \pi / 2$ and, in view of the assumption $V \geq c(|t|+L)^{3}$, also $|a| \leq|z|^{1 / 3}$. Hence from (2.5), (2.6) and $d=1$ we get

$$
h^{*}(w, s) \ll e^{\frac{\pi}{2}(t+V)} \beta^{-|u|}\left(u^{2}+V^{2}\right)^{\frac{1}{2}(|u|+1 / 2-\sigma)} e^{-(V+t) \arctan \frac{V}{|u|}} e^{-|u|}
$$

uniformly for $(\boldsymbol{\lambda}, \boldsymbol{\mu}) \in \mathcal{F}$ and $-L \leq \sigma \leq R$. Moreover, by the same argument we also have

$$
\Gamma(w) \ll \frac{e^{-\pi V}}{|\Gamma(1+|u|-i V)|} \ll e^{-\pi V}\left(u^{2}+V^{2}\right)^{-\frac{1}{2}(|u|+1 / 2)} e^{V \arctan \frac{V}{|u|}} e^{|u|} .
$$

Therefore, recalling that $y>1 / \beta, t \leq c V^{1 / 3}$ and $V \geq c \log ^{-6} \beta y$, substituting $u \rightarrow-u$ we get

$$
\begin{aligned}
J^{+}(V ;-i y, s) & \ll \int_{K+1 / 2}^{\infty}(\beta y)^{-u}\left(u^{2}+V^{2}\right)^{-\sigma / 2} e^{t\left(\frac{\pi}{2}-\arctan \frac{V}{u}\right)} d u \\
& =\left(\int_{K+1 / 2}^{\sqrt{V}}+\int_{\sqrt{V}}^{\infty}\right)(\beta y)^{-u}\left(u^{2}+V^{2}\right)^{-\sigma / 2} e^{t\left(\frac{\pi}{2}-\arctan \frac{V}{u}\right)} d u \\
& \ll \frac{V^{c L} y^{-K-1 / 2}}{\log \beta y}+\frac{V^{c L}}{\log \beta y} e^{\frac{\pi}{2} t-\sqrt{V} \log \beta y} \ll \frac{V^{c L} y^{-K-1 / 2}}{\log \beta y},
\end{aligned}
$$

as required.

Dealing with the $\widetilde{J}^{ \pm}$-integrals, as before we consider only the $\widetilde{J}^{+}$-integral and $t \geq 0$. Writing $w=u+i V$ with $u \geq-K-1 / 2$ and arguing as before, from (2.6) we get

$$
\begin{aligned}
h^{*}(w, s) & \ll e^{-\frac{\pi}{2}(V+t)} \prod_{j=1}^{r}\left|\Gamma\left(\lambda_{j} s+\mu_{j}+\lambda_{j} w\right) \Gamma\left(1-\lambda_{j}(1-s)-\bar{\mu}_{j}+\lambda_{j} w\right)\right|^{-1} \\
& \ll e^{-\frac{\pi}{2}(V+t)} \beta^{u} e^{u}\left(u^{2}+V^{2}\right)^{-\frac{1}{2}(u+\sigma-1 / 2)} e^{(V+t) \arg (u+i V)}
\end{aligned}
$$

uniformly for $(\boldsymbol{\lambda}, \boldsymbol{\mu}) \in \mathcal{F}$ and $-L \leq \sigma \leq R$. Moreover, by Stirling's formula we have

$$
\Gamma(w) \ll\left(u^{2}+V^{2}\right)^{\frac{1}{2}(u-1 / 2)} e^{-V \arg (u+i V)} e^{-u} .
$$

Therefore, recalling that $0<y<1 / \beta$ and observing that $\arg (u+i V) \leq$ 
$\pi / 2+c / V$ for $-K-1 / 2 \leq u<\infty$, we obtain

$$
\begin{aligned}
\widetilde{J}^{+}(V ;-i y, s) & \ll \int_{-K-1 / 2}^{\infty}(\beta y)^{u}\left(u^{2}+V^{2}\right)^{-\sigma / 2} e^{t(\arg (u+i V)-\pi / 2)} d u \\
& \ll\left(\int_{-K-1 / 2}^{\sqrt{V}}+\int_{\sqrt{V}}^{\infty}\right)\left(\frac{1}{\beta y}\right)^{-u}\left(u^{2}+V^{2}\right)^{-\sigma / 2} d u \\
& \ll \frac{V^{c L} y^{-K-1 / 2}}{|\log \beta y|},
\end{aligned}
$$

and the lemma follows.

LEMMA 2.3. Let $\mathcal{F}$ be an admissible family of data, $L \geq 2, y>0$ with $y \neq 1 / \beta, R=R(\mathcal{F}) \geq 2$ and $K$ be as in (2.4). Then for any $(\boldsymbol{\lambda}, \boldsymbol{\mu}) \in \mathcal{F}$ the function $H_{K}(-i y, s)$ is holomorphic for $\sigma \leq R$. Moreover

$$
H_{K}(-i y, s) \ll(|t|+2)^{c L} y^{-K-1 / 2}\left(1+|\log \beta y|^{-c L}\right)
$$

uniformly for $(\boldsymbol{\lambda}, \boldsymbol{\mu}) \in \mathcal{F}$ and $-L \leq \sigma \leq R$, where $c=c(\mathcal{F})$.

Proof. We give only a sketch of proof since the basic argument and the technical details are similar to those in Theorem 4.1 of [7] (see also Lemma 2.1) and in Lemma 2.2, respectively.

Let $(\boldsymbol{\lambda}, \boldsymbol{\mu}) \in \mathcal{F}, V>0$ be sufficiently large and $s$ belong to the rectangle $\mathcal{R}$ defined by $-L \leq \sigma \leq R$ and $V \geq c(|t|+L)^{3}$. Assume first that $y>1 / \beta$ and consider the contour $\mathcal{C}$ consisting of the vertical segment $[-K-1 / 2-i V$, $-K-1 / 2+i V]$ and of the two horizontal half-lines $(-\infty \pm i V,-K-1 / 2 \pm i V]$. Moreover, for $z=-i y$ let

$$
H_{\mathcal{C}}(z, s)=\frac{1}{2 \pi i} \int_{\mathcal{C}} h(w, s) z^{w} d w
$$

Clearly

$$
H_{\mathcal{C}}(-i y, s)=I(V ;-i y, s)+J^{-}(V ;-i y, s)+J^{+}(V ;-i y, s),
$$

where the integrals on the RHS are those in Lemma 2.2, and hence $H_{\mathcal{C}}(-i y, s)$ is holomorphic for $s \in \mathcal{R}$ by Lemma 2.2. Now we apply Cauchy's theorem to the two closed contours obtained by joining the half-lines $(-K-1 / 2 \pm i V$, $-K-1 / 2 \pm i \infty)$ and $(-\infty \pm i V,-K-1 / 2 \pm i V]$ by two arcs of the circle $|w|=U$ with $U \rightarrow \infty$. Note that the region delimited by such closed contours does not contain poles of $h(w, s)(-i y)^{w}$ if $s \in \mathcal{R}$. Moreover, by estimates similar to those in Lemma 2.2 we can show that the integral of $h(w, s)(-i y)^{w}$ over such arcs tends to 0 as $U \rightarrow \infty$. Therefore

$$
H_{\mathcal{C}}(-i y, s)=H_{K}(-i y, s)
$$

and hence $H_{K}(-i y, s)$ is holomorphic for $s \in \mathcal{R}$. Since $L$ and $V$ are arbitrary, 
we see that $H_{K}(-i y, s)$ is holomorphic for $\sigma \leq R$ in this case. Moreover, for $-L \leq \sigma \leq R$ we have

$$
H_{K}(-i y, s)=I(V ;-i y, s)+J^{-}(V ;-i y, s)+J^{+}(V ;-i y, s)
$$

with $V \geq c(|t|+L)^{3}$ and a suitable constant $c=c(\mathcal{F})$.

The case $0<y<1 / \beta$ is dealt with in a similar way, by considering in place of $\mathcal{C}$ the contour $\mathcal{C}^{\prime}$ consisting of the vertical segment $[-K-1 / 2-i V$, $-K-1 / 2+i V]$ and of the two horizontal half-lines $[-K-1 / 2 \pm i V$, $\infty \pm i V)$. Using the integrals $\widetilde{J}^{ \pm}(V ;-i y, s)$ in Lemma 2.2 instead of the corresponding integrals $J^{ \pm}(V ;-i y, s)$, in this case by Cauchy's theorem we deduce that $H_{K}(-i y, s)$ is holomorphic for $\sigma \leq R$ and

$$
H_{K}(-i y, s)=I(V ;-i y, s)+\widetilde{J}^{-}(V ;-i y, s)+\widetilde{J}^{+}(V ;-i y, s)
$$

for $-L \leq \sigma \leq R$ and $V \geq c(|t|+L)^{3}$.

In order to get a uniform bound for $H_{K}(-i y, s)$ in the strip $-L \leq \sigma \leq R$ we inject the bounds of Lemma 2.2 into (2.7) and (2.8). Suppose first that $y>1 / \beta$. If $|t| \gg \log ^{-2} \beta y$ we choose $V=c(|t|+L)^{3} \gg \log ^{-6} \beta y$ in (2.7), and hence from Lemma 2.2 we get

$$
H_{K}(-i y, s) \ll(|t|+2)^{c L} y^{-K-1 / 2}\left(1+\frac{1}{\log \beta y}\right) .
$$

If $|t| \ll \log ^{-2} \beta y$ we choose $V=c\left(L^{3}+\log ^{-6} \beta y\right) \gg(|t|+L)^{3}$ in $(2.7)$, and hence from Lemma 2.2 we have

$$
H_{K}(-i y, s) \ll(\log \beta y)^{-c L} y^{-K-1 / 2}\left(1+\frac{1}{\log \beta y}\right) .
$$

Therefore, for $y>1 / \beta$ we have

$$
H_{K}(-i y, s) \ll(|t|+2)^{c L} y^{-K-1 / 2}\left(1+\log ^{-c L} \beta y\right)
$$

uniformly for $(\boldsymbol{\lambda}, \boldsymbol{\mu}) \in \mathcal{F}$ and $-L \leq \sigma \leq R$. A similar (even simpler) argument applies to the case $0<y<1 / \beta$ as well, and the lemma follows.

We recall that for $F \in \mathcal{S}^{\sharp}$ the $\xi$-invariant $\xi_{F}$ (see the Introduction) is defined in terms of the $\boldsymbol{\mu}$-data. In analogy with $\xi_{F}$, starting from the data $(\boldsymbol{\lambda}, \boldsymbol{\mu})$ we define

$$
\xi=2 \sum_{j=1}^{r}\left(\mu_{j}-1 / 2\right)=\eta+i \theta
$$

and write

$$
A(s)=\sum_{j=1}^{r}\left(\lambda_{j}(1-2 s)-2 i \Im \mu_{j}\right) \log 2 \lambda_{j}
$$

and, for any non-negative integer $m$, 


$$
\begin{aligned}
\Gamma_{K, m}(s)= & -\sum_{k=0}^{K} \frac{1}{k !} \frac{\Gamma\left(1-\frac{s}{2}+\frac{\bar{\xi}}{2}+\frac{k}{2}\right)}{\Gamma\left(\frac{1}{2}+\frac{s}{2}+\frac{\xi}{2}-\frac{k}{2}+m\right)}\left(\frac{i}{2}\right)^{-k} \\
& +\sqrt{\pi} \frac{\Gamma\left(1-\frac{s}{2}+\frac{\bar{\xi}}{2}\right) \Gamma(s-1+i \theta+m)}{\Gamma\left(\frac{s}{2}-\frac{1}{2}-\frac{\bar{\xi}}{2}\right) \Gamma\left(\frac{s}{2}+\frac{\xi}{2}+m\right) \Gamma\left(\frac{1}{2}+\frac{s}{2}+\frac{\xi}{2}+m\right)} \\
& -i \sqrt{\pi} \frac{\Gamma\left(\frac{3}{2}-\frac{s}{2}+\frac{\bar{\xi}}{2}\right) \Gamma(s-1+i \theta+m)}{\Gamma\left(\frac{s}{2}-\frac{\bar{\xi}}{2}\right) \Gamma\left(\frac{s}{2}+\frac{\xi}{2}+m\right) \Gamma\left(\frac{1}{2}+\frac{s}{2}+\frac{\xi}{2}+m\right)} .
\end{aligned}
$$

Lemma 2.4. Let $\mathcal{F}$ be an admissible family of data, $L \geq 2, R=R(\mathcal{F}) \geq 2$, $K$ be as in (2.4) and $\xi$ be as in (2.9). Then for any $(\boldsymbol{\lambda}, \boldsymbol{\mu}) \in \mathcal{F}$ and any integer $M \geq c L$ with a suitable $c=c(\mathcal{F})$ we have

$$
H_{K}(-i / \beta, s)=e^{A(s)} \sum_{m=0}^{M} P_{m}(s) \Gamma_{K, m}(s)+g_{M}(s),
$$

where the $P_{m}(s)$ 's are polynomials with $\operatorname{deg} P_{m} \leq 2 m$ and $P_{0}(s)=1$ identically, and their coefficients are uniformly bounded by a constant depending of $\mathcal{F}$ and $M$. Moreover, $g_{M}(s)$ is holomorphic for $-L \leq \sigma \leq R$ and satisfies

$$
g_{M}(s) \ll \mathcal{F}, M(|t|+2)^{c M}
$$

uniformly for $(\boldsymbol{\lambda}, \boldsymbol{\mu}) \in \mathcal{F}$ and $-L \leq \sigma \leq R$, where $c=c(\mathcal{F})$.

Proof. This lemma is an extension of Theorem 5.1 in [7], and the main argument in the proof is similar. Therefore, we only give a sketch of the proof.

Let $\mathcal{F}$ be an admissible family of data, $(\boldsymbol{\lambda}, \boldsymbol{\mu}) \in \mathcal{F}, z=x-i / \beta$ with $x>0$ and $w=-K-1 / 2+i v$. Then for $V>0$ sufficiently large we have

$$
\begin{aligned}
H_{K}(z, s) & =\frac{1}{2 \pi i}\left(\int_{|v| \leq V}+\int_{|v| \geq V}\right) h(w, s) z^{w} d w \\
& =H_{K, V}^{(1)}(z, s)+H_{K, V}^{(2)}(z, s),
\end{aligned}
$$

say. Moreover

$$
H_{K}(-i / \beta, s)=\lim _{x \rightarrow 0^{+}} H_{K}(z, s) .
$$

Since $H_{K, V}^{(1)}(z, s)$ is the integral $I(V ; z, s)$ in Lemma 2.2, we infer that $H_{K, V}^{(1)}(-i / \beta, s)$ is holomorphic for $\sigma \leq R$. Moreover, we choose $V=c(|t|+L)^{3}$ with a suitable constant $c=c(\mathcal{F})$ and apply Lemma 2.2, thus getting (recall that the value of $c$ is not necessarily the same at each occurrence)

$$
H_{K, V}^{(1)}(-i / \beta, s) \ll(|t|+2)^{c L}
$$

uniformly for $(\boldsymbol{\lambda}, \boldsymbol{\mu}) \in \mathcal{F}$ and $-L \leq \sigma \leq R$. 
In order to deal with $H_{K, V}^{(2)}(z, s)$ we write $a=\frac{1}{2}(1-s)+\frac{1}{2} \bar{\xi}$ and $b=$ $\frac{1}{2} s+\frac{1}{2} \xi$, and argue as in Lemma 5.1 of [7] using the following form of Stirling's formula:

$$
\begin{aligned}
\log \Gamma(z+a)= & \left(z+a-\frac{1}{2}\right) \log z-z+\frac{1}{2} \log 2 \pi \\
& +\sum_{m=1}^{M}(-1)^{m+1} \frac{B_{m+1}(a)}{m(m+1)} z^{-m}+O\left(\frac{|a|^{M+2}}{|z|^{M+1}}\right)
\end{aligned}
$$

uniformly for $|\arg (z+a)| \leq \pi-\varepsilon$ and $|a| \leq|z| / 2$, where $M$ is any nonnegative integer and $B_{m}(a)$ denotes the $m$ th Bernoulli polynomial. Applying this to $h^{*}(w, s)$ we get

$\log h^{*}(w, s)=\log \left\{e^{A(s)} \frac{\Gamma\left(a+\frac{1}{2}-\frac{w}{2}\right)}{\Gamma\left(b+\frac{1}{2}+\frac{w}{2}\right)}\left(\frac{\beta}{2}\right)^{w}\right\}+\sum_{m=1}^{M} \frac{Q_{m}(s)}{w^{m}}+O\left(\frac{|s|^{M+2}}{|w|^{M+1}}\right)$

with certain polynomials $Q_{m}(s)$ of degree $\leq m+1$, and taking the exponential we obtain

$$
h^{*}(w, s)=e^{A(s)} \frac{\Gamma\left(a+\frac{1}{2}-\frac{w}{2}\right)}{\Gamma\left(b+\frac{1}{2}+\frac{w}{2}\right)}\left(\frac{\beta}{2}\right)^{w}\left\{1+\sum_{m=1}^{M} \frac{Q_{m}^{\prime}(s)}{w^{m}}+O\left(\frac{|s|^{2 M+2}}{|w|^{M+1}}\right)\right\}
$$

with certain polynomials $Q_{m}^{\prime}(s)$ of degree $\leq 2 m$. Now for $1 \leq m \leq M$ we use the formula

$$
\frac{1}{w^{m}}=\frac{\alpha^{m}}{(\alpha w+\beta)_{m}}+\sum_{l=1}^{M-m-1} \frac{F_{l, m}(\beta)}{w^{l+m}}+O\left(\frac{|\beta|^{M-m}}{|w|^{M}}\right)
$$

with certain polynomials $F_{l, m}(\beta)$ of degree $\leq l$, where $\alpha$ and $\beta$ are parameters to be suitably chosen and $(z)_{m}$ means 1 for $m=0$ and $z(z+1) \cdots$ $\cdots(z+m-1)$ for $m \geq 1$. In this way we finally get

$$
h^{*}(w, s)=e^{A(s)} \frac{\Gamma\left(a+\frac{1}{2}-\frac{w}{2}\right)}{\Gamma\left(b+\frac{1}{2}+\frac{w}{2}\right)}\left(\frac{\beta}{2}\right)^{w}\left(\sum_{m=0}^{M} \frac{P_{m}(s)}{\left(b+\frac{1}{2}+\frac{w}{2}\right)_{m}}+g_{M}(w, s)\right),
$$

where the $P_{m}(s)$ 's are polynomials with $\operatorname{deg} P_{m} \leq 2 m$ and $P_{0}(s)=1$ identically, and their coefficients are uniformly bounded by a constant depending on $\mathcal{F}$ and $M$. Moreover, if we choose $M \geq c L$ with a suitable constant $c=c(\mathcal{F})$, then $g_{M}(w, s)$ is holomorphic for $-L \leq \sigma \leq R$ and satisfies

$$
g_{M}(w, s) \ll \frac{(|t|+2)^{M+2}}{|v|^{M+1}}, \quad|v| \geq V
$$

uniformly for $(\boldsymbol{\lambda}, \boldsymbol{\mu}) \in \mathcal{F}$ and $-L \leq \sigma \leq R$. Hence, using $\Gamma(z+1)=z \Gamma(z)$, we get 
(2.13) $\quad H_{K, V}^{(2)}(z, s)$

$$
=e^{A(s)} \sum_{m=0}^{M} \frac{P_{m}(s)}{2 \pi i} \int_{(-K-1 / 2)} \frac{\Gamma\left(a+\frac{1}{2}-\frac{w}{2}\right) \Gamma(w)}{\Gamma\left(b+\frac{1}{2}+\frac{w}{2}+m\right)}\left(\frac{\beta z}{2}\right)^{w} d w+\widetilde{H}_{K, V, M}^{(2)}(z, s),
$$

where

$$
\begin{aligned}
\widetilde{H}_{K, V, M}^{(2)}(z, s)= & -e^{A(s)} \sum_{m=0}^{M} \frac{P_{m}(s)}{2 \pi i} \int_{|v| \leq V} \frac{\Gamma\left(a+\frac{1}{2}-\frac{w}{2}\right) \Gamma(w)}{\Gamma\left(b+\frac{1}{2}+\frac{w}{2}+m\right)}\left(\frac{\beta z}{2}\right)^{w} d w \\
& +e^{A(s)} \frac{1}{2 \pi i} \int_{|v| \geq V} \frac{\Gamma\left(a+\frac{1}{2}-\frac{w}{2}\right) \Gamma(w)}{\Gamma\left(b+\frac{1}{2}+\frac{w}{2}\right)}\left(\frac{\beta z}{2}\right)^{w} g_{M}(w, s) d w .
\end{aligned}
$$

In view of the choice of $M$ the second integral is convergent in the strip $-L \leq \sigma \leq R$, and hence $\widetilde{H}_{K, V, M}^{(2)}(-i / \beta, s)$ is holomorphic in the same strip and satisfies

$$
\widetilde{H}_{K, V, M}^{(2)}(-i / \beta, s) \ll(|t|+2)^{c M}
$$

uniformly for $(\boldsymbol{\lambda}, \boldsymbol{\mu}) \in \mathcal{F}$ and $-L \leq \sigma \leq R$.

In order to deal with the first term on the RHS of (2.13) we observe that each term

$$
\frac{e^{A(s)}}{2 \pi i} \int_{(-K-1 / 2)} \frac{\Gamma\left(a+\frac{1}{2}-\frac{w}{2}\right) \Gamma(w)}{\Gamma\left(b+\frac{1}{2}+\frac{w}{2}+m\right)}\left(\frac{\beta z}{2}\right)^{w} d w
$$

equals the function $H_{K}^{(1)}(z, s)$ in (5.2) of [7] with $b$ replaced by $b+m$. Therefore, arguing exactly as in the proof of Theorem 5.1 of [7] we get

$$
\begin{aligned}
\lim _{x \rightarrow 0^{+}} e^{A(s)} \sum_{m=0}^{M} \frac{P_{m}(s)}{2 \pi i} \int_{(-K-1 / 2)} \frac{\Gamma\left(a+\frac{1}{2}-\frac{w}{2}\right) \Gamma(w)}{\Gamma\left(b+\frac{1}{2}+\frac{w}{2}+m\right)}\left(\frac{\beta z}{2}\right)^{w} d w \\
=e^{A(s)} \sum_{m=0}^{M} P_{m}(s)\left\{-\sum_{k=0}^{K} \frac{1}{k !} \frac{\Gamma\left(a+\frac{1}{2}+\frac{k}{2}\right)}{\Gamma\left(b+\frac{1}{2}-\frac{k}{2}+m\right)}\left(\frac{i}{2}\right)^{-k}\right. \\
+\sqrt{\pi} \frac{\Gamma\left(a+\frac{1}{2}\right) \Gamma\left(b-a-\frac{1}{2}+m\right)}{\Gamma(-a) \Gamma(b+m) \Gamma\left(\frac{1}{2}+b+m\right)} \\
\left.-i \sqrt{\pi} \frac{\Gamma(a+1) \Gamma\left(b-a-\frac{1}{2}+m\right)}{\Gamma\left(\frac{1}{2}-a\right) \Gamma(b+m) \Gamma\left(\frac{1}{2}+b+m\right)}\right\}
\end{aligned}
$$

In view of the definitions of $a$ and $b$, the lemma follows now from (2.10)-(2.15).

Lemma 2.5. Let $\mathcal{F}$ be an admissible family of data, $L \geq 2, R=R(\mathcal{F}) \geq 2$, $K$ be as in (2.4) and $\theta$ be as in (2.9). Then for any $(\boldsymbol{\lambda}, \boldsymbol{\mu}) \in \mathcal{F}$ the function $H_{K}(-i / \beta, s)$ is meromorphic for $\sigma \leq R$, with at most simple poles at the 
points

$$
s^{(k)}=1-k-i \theta, \quad k=0,1, \ldots,
$$

and non-vanishing residue at $s^{(0)}$. Moreover, for $\left|s-s^{(k)}\right| \geq c, k=0,1, \ldots$,

$$
H_{K}(-i / \beta, s) \ll \mathcal{F}, L(|t|+2)^{c L}
$$

uniformly for $(\boldsymbol{\lambda}, \boldsymbol{\mu}) \in \mathcal{F}$ and $-L \leq \sigma \leq R$, where $c=c(\mathcal{F})$.

Proof. This lemma is an extension of Theorem 6.1 of [7], but the argument in the proof is different. In fact, the argument using binomial coefficient identities as in [7] is apparently difficult to carry over to our present case, and is replaced by a more theoretical argument.

Let $(\boldsymbol{\lambda}, \boldsymbol{\mu}) \in \mathcal{F}$. We choose $M=[c L]+1$ in Lemma 2.4 to infer that $H_{K}(-i / \beta, s)$ is meromorphic for $\sigma \leq R$ since $L$ can be taken arbitrarily large. Moreover, by Lemma 2.4 and Stirling's formula we find that $H_{K}(-i / \beta, s)$ satisfies the required bound away from its poles. Therefore, the proof of the lemma reduces to investigating the polar structure of $H_{K}(-i / \beta, s)$.

We start by observing that for $a, b, c, d \in \mathbb{C}$ with $a c \neq 0$ and $w$ with $(a-c) w+b-d$ non-negative integer, the function

$$
\frac{\Gamma(a s+b)}{\Gamma(c s+d)}
$$

is holomorphic at $s=w$. In fact, denoting by $f(s)$ the function in $(2.16)$ and writing $k=(a-c) w+b-d$, we have

$$
f(s)=\frac{\Gamma(a(s-w)+a w+b)}{\Gamma(c(s-w)+c w+d)}=Q_{k}(s) \frac{\Gamma\left(a(s-w)+z_{0}\right)}{\Gamma\left(c(s-w)+z_{0}\right)},
$$

where $Q_{k}(s)$ is a polynomial of degree $k$ and $z_{0}=c w+d$, and our assertion follows at once.

Next we consider the meromorphic function $\Gamma_{K, m}(s)$ as in Lemma 2.4, and prove that for every $m \geq 0$ it has at most simple poles. In fact, if we write

$$
\Gamma_{K, m}(s)=-\Sigma(s)+\sqrt{\pi} A(s)-i \sqrt{\pi} B(s),
$$

then $\Sigma(s)$ has obviously at most simple poles, while the possible poles of $A(s)$ and $B(s)$ are at the points

$$
w_{l}=\bar{\xi}+l
$$

with $l$ integer $\geq 2$, coming from $\Gamma\left(1-\frac{s}{2}+\frac{\bar{\xi}}{2}\right)$ and $\Gamma\left(\frac{3}{2}-\frac{s}{2}+\frac{\bar{\xi}}{2}\right)$, and

$$
s^{(k)}=1-k-i \theta
$$

with $k$ integer $\geq m$, coming from $\Gamma(s-1+i \theta+m)$. Moreover, $A(s)$ and $B(s)$ have at most double poles, and this may a priori happen only when 
$w_{l}=s^{(k)}$ for some $l$ and $k$ in the above ranges. But if we write

$$
f_{1}(s)=\frac{\Gamma\left(1-\frac{s}{2}+\frac{\bar{\xi}}{2}\right)}{\Gamma\left(\frac{s}{2}+\frac{\xi}{2}+m\right)}, \quad f_{2}(s)=\frac{\Gamma\left(\frac{3}{2}-\frac{s}{2}+\frac{\bar{\xi}}{2}\right)}{\Gamma\left(\frac{1}{2}+\frac{s}{2}+\frac{\xi}{2}+m\right)},
$$

then for $w=s^{(k)}$ the functions $f_{1}(s)$ and $f_{2}(s)$ satisfy the hypotheses of (2.16) and hence are holomorphic at $s=s^{(k)}$. Therefore, $A(s)$ and $B(s)$ have at most simple poles as well, thus proving our assertion. In particular, $H_{K}(-i / \beta, s)$ has at most simple poles.

Writing $w=-K-1 / 2+i v$ and $z=x-i / \beta$ with $x>0$, from (2.15) we have

$$
\Gamma_{K, m}(s)=\lim _{x \rightarrow 0^{+}} \frac{1}{2 \pi i} \int_{(-K-1 / 2)} \frac{\Gamma\left(1-\frac{s}{2}+\frac{\bar{\xi}}{2}-\frac{w}{2}\right) \Gamma(w)}{\Gamma\left(\frac{1}{2}+\frac{s}{2}+\frac{\xi}{2}+\frac{w}{2}+m\right)}\left(\frac{\beta z}{2}\right)^{w} d w,
$$

and by Stirling's formula

$$
\frac{\Gamma\left(1-\frac{s}{2}+\frac{\bar{\xi}}{2}-\frac{w}{2}\right) \Gamma(w)}{\Gamma\left(\frac{1}{2}+\frac{s}{2}+\frac{\xi}{2}+\frac{w}{2}+m\right)}\left(\frac{\beta z}{2}\right)^{w} \ll(|v|+2)^{-\sigma-m}
$$

uniformly for $x \rightarrow 0^{+}$. Therefore, $\Gamma_{K, m}(s)$ is holomorphic for $1-m<\sigma$ $\leq R$, and hence by Lemma 2.4 the function $H_{K}(-i / \beta, s)$ is holomorphic for $1<\sigma \leq R$.

Now we are ready to conclude the proof of the lemma. Note that the possible poles of $\Sigma(s)$ in (2.17) are of type $w_{l}$ (see (2.18)), and hence by Lemma 2.4 the possible poles of $H_{K}(-i / \beta, s)$ in the half-plane $\sigma \leq 1$ are of type $w_{l}$ or $s^{(k)}$ (see (2.18) and (2.19)). Let $w_{l}$ be such that $\Re w_{l} \leq 1$ and $w_{l} \neq s^{(k)}$ for every $k \geq 0$. Since the functions $\Gamma_{K, m}(s)$ have at most simple poles, in order to prove that $H_{K}(-i / \beta, s)$ is holomorphic at $s=w_{l}$ it suffices to show that

$$
\operatorname{res}_{s=w_{l}} \Gamma_{K, m}(s)=0, \quad m \geq 0 .
$$

Suppose first that $l$ is even, say $l=2 p$. Then, since $w_{l} \neq s^{(k)}, B(s)$ in $(2.17)$ is holomorphic at $s=w_{l}$. Moreover, $\Gamma\left(1-\frac{s}{2}+\frac{\bar{\xi}}{2}+\frac{k}{2}\right)$ is also holomorphic at $s=w_{l}$ if $k$ is odd or if $k=2 q$ with integer $q>p-1$. Therefore by (2.17) we get

$$
\begin{aligned}
& \operatorname{res}_{s=w_{l}} \Gamma_{K, m}(s) \\
&=-\sum_{q=0}^{p-1} \frac{1}{(2 q) !}\left(\frac{i}{2}\right)^{-2 q} \frac{1}{\Gamma\left(\frac{1}{2}+\frac{w_{l}}{2}+\frac{\xi}{2}-q+m\right)} \lambda_{p, q} \\
&+\sqrt{\pi} \frac{\Gamma\left(w_{l}-1+i \theta+m\right)}{\Gamma\left(\frac{w_{l}}{2}-\frac{1}{2}-\frac{\bar{\xi}}{2}\right) \Gamma\left(\frac{w_{l}}{2}+\frac{\xi}{2}+m\right) \Gamma\left(\frac{1}{2}+\frac{w_{l}}{2}+\frac{\xi}{2}+m\right)} \lambda_{p, 0},
\end{aligned}
$$


where for $q \leq p-1$

$\lambda_{p, q}=\operatorname{res}_{s=w_{2 p}} \Gamma\left(1-\frac{s}{2}+\frac{\bar{\xi}}{2}+q\right)=-2 \operatorname{res}_{z=1-p+q} \Gamma(z)=-2 \frac{(-1)^{p-1-q}}{(p-1-q) !}$,

and hence

$$
\begin{aligned}
\operatorname{res}_{s=} w_{2 p} & \Gamma_{K, m}(s) \\
= & 2(-1)^{p-1}\left\{\sum_{q=0}^{p-1} \frac{4^{q}}{(2 q) !(p-1-q) ! \Gamma\left(\frac{1}{2}+p-q+\eta+m\right)}\right. \\
& \left.-\sqrt{\pi} \frac{\Gamma(2 p-1+\eta+m)}{(p-1) ! \Gamma\left(p-\frac{1}{2}\right) \Gamma(p+\eta+m) \Gamma\left(\frac{1}{2}+p+\eta+m\right)}\right\} \\
= & 2(-1)^{p-1} \Phi_{p}(\eta+m),
\end{aligned}
$$

say. The function $\Phi_{p}(z)$ is clearly meromorphic for every $p$, and $\Phi_{p}(x)=0$ for $1-2 p<x \leq R-2 p$. Indeed, given $x$ in such an interval we choose $(\boldsymbol{\lambda}, \boldsymbol{\mu})$ with $\lambda_{j}>0, d=1$ and $\eta=\Re \xi=x$, and consider the associated functions $\Gamma_{K, m}(s)$ and the point $w_{2 p}=\bar{\xi}+2 p$. Since in this case $1<\Re w_{2 p} \leq R$, we already know that $\Gamma_{K, 0}(s)$ is holomorphic at $s=w_{2 p}$, and hence $\Phi_{p}(x)=0$ by (2.22). By analytic continuation we have $\Phi_{p}(z)=0$ identically, therefore (2.20) follows from (2.22) in the case of even $l$.

The case of odd $l$ can be treated in a completely analogous way, with the role of $A(s)$ in the second term on the RHS of (2.21) replaced by $B(s)$. In such a way we find that $H_{K}(-i / \beta, s)$ is holomorphic at the points $w_{l}$ in (2.18), provided $w_{l} \neq s^{(k)}$. The singularities of $H_{K}(-i / \beta, s)$ with $\sigma \leq R$ are therefore at most simple poles at the points $s^{(k)}$ in (2.19) with $k \geq 0$. Moreover, the argument leading to Theorem 6.1 of [7] shows that the residue of $\Gamma_{K, 0}(s)$ at $s=s^{(0)}$ does not vanish, while we already know that the functions $\Gamma_{K, m}(s)$ with $m \geq 1$ are holomorphic at $s=s^{(0)}$. Since $P_{0}(s)=1$ identically, by Lemma 2.4 we conclude that the residue of $H_{K}(-i / \beta, s)$ at $s=s^{(0)}$ does not vanish, and the lemma follows.

By Lemmas 2.3 and 2.5 we summarize the required properties of $H_{K}(-i y, s)$ as follows. Writing

$$
\chi(y)= \begin{cases}1 & \text { if } y \neq 1 / \beta \\ 0 & \text { if } y=1 / \beta\end{cases}
$$

we have

THEOREM 2.1. Let $\mathcal{F}$ be an admissible family of data, $L \geq 2, y>0, R=$ $R(\mathcal{F}) \geq 2, K$ be as in (2.4) and $\theta$ be as in (2.9). Then for any $(\boldsymbol{\lambda}, \boldsymbol{\mu}) \in \mathcal{F}$ the function $H_{K}(-i y, s)$ is meromorphic for $\sigma \leq R$. Moreover 
(i) if $y \neq 1 / \beta$ then $H_{K}(-i y, s)$ is holomorphic for $\sigma \leq R$, while $H_{K}(-i / \beta, s)$ has at most simple poles at the points

$$
s^{(k)}=1-k-i \theta, \quad k=0,1, \ldots,
$$

with non-vanishing residue at $s^{(0)}$;

(ii) we have $\left(\right.$ for $\left|s-s^{(k)}\right| \geq c, k=0,1, \ldots$, if $\left.y=1 / \beta\right)$

$$
H_{K}(-i y, s) \ll \mathcal{F}, L(|t|+2)^{c L} y^{-K-1 / 2}\left(1+\chi(y)|\log \beta y|^{-c L}\right)
$$

uniformly for $(\boldsymbol{\lambda}, \boldsymbol{\mu}) \in \mathcal{F}$ and $-L \leq \sigma \leq R$, where $c=c(\mathcal{F})$.

3. Proof of Theorems 1 and 2. The proof of Theorem 1 follows the proof of Theorem 7.1 of [7], and hence we give a sketch of the main argument, with details where needed. Let $d>0, F \in \mathcal{S}_{d}^{\sharp}$ and $\alpha>0$. Moreover, let $\Delta \geq 2, R \geq 2$ to be chosen later on, $K$ be as in (2.4), $N>2$ and $z_{N}=$ $1 / N+2 \pi i \alpha$. For $\sigma<1+1 / d$ and a sufficiently large constant $c>0$, the argument at the beginning of Section 7 of [7] and transformation (2.3) give

$$
\begin{aligned}
& F_{N}(s, \alpha) \\
& =\sum_{n=1}^{\infty} \frac{a(n)}{n^{s}} e^{-n^{1 / d} z_{N}}=\frac{1}{2 \pi i} \int_{(c)} F\left(s+\frac{w}{d}\right) \Gamma(w) z_{N}^{-w} d w \\
& =R_{N}^{(1)}(s, \alpha)+R_{N}^{(2)}(s, \alpha)+\omega Q^{1-2 s} \sum_{n=1}^{\infty} \frac{\overline{a(n)}}{n^{1-s}} H_{K}\left(\frac{n^{1 / d}}{Q^{2 / d} z_{N}}, s ; \boldsymbol{\lambda}, \boldsymbol{\mu}\right) \\
& =R_{N}^{(1)}(s, \alpha)+R_{N}^{(2)}(s, \alpha)+\omega Q^{1-2 s} \sum_{n=1}^{\infty} \frac{\overline{a(n)}}{n^{1-s}} H_{K}\left(\frac{n^{1 / d}}{Q^{2 / d} z_{N}}, \widetilde{s} ; \tilde{\boldsymbol{\lambda}}, \tilde{\boldsymbol{\mu}}\right),
\end{aligned}
$$

where $H_{K}(z, s ; \boldsymbol{\lambda}, \boldsymbol{\mu})$ is the hypergeometric function defined by $(2.1), \widetilde{s}, \widetilde{\boldsymbol{\lambda}}, \widetilde{\boldsymbol{\mu}}$ are given by $(2.2)$ and

$$
\begin{aligned}
& R_{N}^{(1)}(s, \alpha)=\operatorname{res}_{w=d(1-s)} F\left(s+\frac{w}{d}\right) \Gamma(w) z_{N}^{-w}, \\
& R_{N}^{(2)}(s, \alpha)=\sum_{k=0}^{K} \frac{(-1)^{k}}{k !} F\left(s-\frac{k}{d}\right) z_{N}^{k} .
\end{aligned}
$$

Letting $N \rightarrow \infty$, from (3.1) and the definition of $n_{\alpha}$ we obtain

$$
\begin{aligned}
F(s, \alpha)= & R^{(1)}(s, \alpha)+R^{(2)}(s, \alpha) \\
& +\omega Q^{1-2 s} \sum_{n=1}^{\infty} \frac{\overline{a(n)}}{n^{1-s}} H_{K}\left(-\frac{i}{d \beta^{1 / d}}\left(\frac{n}{n_{\alpha}}\right)^{1 / d}, \widetilde{s} ; \widetilde{\boldsymbol{\lambda}}, \widetilde{\boldsymbol{\mu}}\right),
\end{aligned}
$$

where

$$
R^{(j)}(s, \alpha)=\lim _{N \rightarrow \infty} R_{N}^{(j)}(s, \alpha), \quad j=1,2 .
$$


In order to deal with the first two terms on the RHS of (3.2), we write the Laurent expansion of $F(s)$ at $s=1$ as

$$
F(s)=\sum_{k=1}^{m_{F}} \frac{\alpha_{k}}{(s-1)^{k}}+f_{1}(s)
$$

with $f_{1}(s)$ entire. A computation shows that

$$
R_{N}^{(1)}(s, \alpha)=\sum_{k=1}^{m_{F}} \alpha_{k} d^{k} \beta_{k-1, N}(s)
$$

with

$$
\beta_{k, N}(s)=\frac{1}{k !} \sum_{\nu=0}^{k}\left(\begin{array}{l}
k \\
\nu
\end{array}\right) \Gamma^{(\nu)}((1-s) d)\left(-\log z_{N}\right)^{k-\nu} z_{N}^{(s-1) d},
$$

and hence

$$
R^{(1)}(s, \alpha)=\sum_{k=1}^{m_{F}} \alpha_{k} d^{k} \beta_{k-1}(s)
$$

with

$$
\beta_{k}(s)=\frac{1}{k !} \sum_{\nu=0}^{k}\left(\begin{array}{l}
k \\
\nu
\end{array}\right) \Gamma^{(\nu)}((1-s) d)(-\log z)^{k-\nu} z^{(s-1) d}
$$

and $z=2 \pi i \alpha$. Therefore, $R^{(1)}(s, \alpha)$ is holomorphic for $\sigma<1+1 / d, s \neq 1$. Moreover, from the behavior of $\Gamma^{(\nu)}(s)$ at $s=0$ we obtain

$$
\begin{aligned}
\beta_{k}(s) & =\frac{z^{(s-1) d}}{k !} \sum_{\nu=0}^{k}\left(\begin{array}{l}
k \\
\nu
\end{array}\right) \frac{(-1)^{\nu} \nu !}{((1-s) d)^{\nu+1}}(-\log z)^{k-\nu}+f_{2}(s) \\
& =-\frac{z^{(s-1) d}}{k !} \sum_{\nu=0}^{k}\left(\begin{array}{l}
k \\
\nu
\end{array}\right) \frac{(-1)^{k-\nu+1}(k-\nu) !}{((1-s) d)^{k-\nu+1}}(-\log z)^{\nu}+f_{2}(s) \\
& =-\frac{z^{(s-1) d}}{((s-1) d)^{k+1}} \sum_{\nu=0}^{k} \frac{1}{\nu !}(-(s-1) d \log z)^{\nu}+f_{2}(s) \\
& =-\frac{z^{(s-1) d}}{((s-1) d)^{k+1}} \sum_{\nu=0}^{\infty} \frac{1}{\nu !}(-(s-1) d \log z)^{\nu}+f_{3}(s) \\
& =-\frac{1}{((s-1) d)^{k+1}}+f_{3}(s)
\end{aligned}
$$

where $f_{2}(s), f_{3}(s)$ are holomorphic at $s=1$. Hence

$$
R^{(1)}(s, \alpha)=-\sum_{k=1}^{m_{F}} \frac{\alpha_{k}}{(s-1)^{k}}+f_{4}(s)
$$


with $f_{4}(s)$ holomorphic for $\sigma<1+1 / d$. But

$$
R^{(2)}(s, \alpha)=\sum_{k=0}^{K} \frac{(-1)^{k}}{k !} F\left(s-\frac{k}{d}\right) z^{k}
$$

is holomorphic for $\sigma<1+1 / d$ apart from the term corresponding to $k=0$, therefore from (3.3) and (3.6) we see that $R^{(1)}(s, \alpha)+R^{(2)}(s, \alpha)$ is holomorphic for $\sigma<1+1 / d$.

Given $F \in \mathcal{S}_{d}^{\sharp}$ with $d>0$, let $\mathcal{F}$ denote the admissible family of data consisting only of $(\widetilde{\boldsymbol{\lambda}}, \widetilde{\boldsymbol{\mu}})$. We apply Theorem 2.1 to such $\mathcal{F}$, with the choice

$$
R=2 d+2, \quad L=d(\Delta+1)+2 .
$$

Note that, by (2.2), the range of $\sigma$ as $-L \leq \Re \widetilde{s} \leq R$ contains the interval $[-\Delta, 1+1 / d$ ). Note also that the $\beta$-parameter (defined by $(2.5)$ ) of $H_{K}(z, \widetilde{s} ; \widetilde{\boldsymbol{\lambda}}, \widetilde{\boldsymbol{\mu}})$ is

$$
\widetilde{\beta}=d \beta^{1 / d},
$$

while the $\theta$-invariant (see $(2.9)$ ) of $H_{K}\left(z, \widetilde{s} ; \widetilde{\boldsymbol{\lambda}}, \widetilde{\boldsymbol{\mu}}\right.$ ) equals $\theta_{F}$ since $\Im \widetilde{\mu}_{j}=\Im \mu_{j}$. Splitting the sum over $n$ in (3.2) as

$$
\frac{\overline{a\left(n_{\alpha}\right)}}{n_{\alpha}^{1-s}} H_{K}\left(-\frac{i}{\widetilde{\beta}}, \widetilde{s} ; \widetilde{\boldsymbol{\lambda}}, \widetilde{\boldsymbol{\mu}}\right)+\sum_{n \neq n_{\alpha}} \frac{\overline{a(n)}}{n^{1-s}} H_{K}\left(-\frac{i}{\widetilde{\beta}}\left(\frac{n}{n_{\alpha}}\right)^{1 / d}, \widetilde{s} ; \widetilde{\boldsymbol{\lambda}}, \tilde{\boldsymbol{\mu}}\right),
$$

from Theorem 2.1(ii) we have

$$
H_{K}\left(-\frac{i}{\widetilde{\beta}}\left(\frac{n}{n_{\alpha}}\right)^{1 / d}, \widetilde{s} ; \tilde{\boldsymbol{\lambda}}, \widetilde{\boldsymbol{\mu}}\right) \ll n^{-(K+1 / 2) / d} \ll n^{-R / d}, \quad n \rightarrow \infty,
$$

uniformly for $-\Delta \leq \sigma<1+1 / d$, and hence by Theorem 2.1(i) the second term in (3.9) is holomorphic for $\sigma<1+1 / d$ since $n \neq n_{\alpha}$. Moreover, if $a\left(n_{\alpha}\right) \neq 0$ then by Theorem 2.1(i) the first term in (3.9) has at most simple poles at the points $\widetilde{s}^{(k)}=1-k-i \theta_{F}$ with integer $k \geq 0$ and non-vanishing residue at $\widetilde{s}^{(0)}$. In view of $(2.2)$ we therefore deduce that

$$
\omega Q^{1-2 s} \sum_{n=1}^{\infty} \frac{\overline{a(n)}}{n^{1-s}} H_{K}\left(-\frac{i}{d \beta^{1 / d}}\left(\frac{n}{n_{\alpha}}\right)^{1 / d}, \widetilde{s} ; \tilde{\boldsymbol{\lambda}}, \tilde{\boldsymbol{\mu}}\right)
$$

is meromorphic for $\sigma<1+1 / d$, is holomorphic for $\sigma<1+1 / d$ if $a\left(n_{\alpha}\right)=0$, and has at most simple poles at the points

$$
s_{k}=\frac{d+1}{2 d}-\frac{k}{d}-i \frac{\theta_{F}}{d}, \quad k=0,1, \ldots,
$$

if $a\left(n_{\alpha}\right) \neq 0$, with non-vanishing residue at $s_{0}$. This completes the proof of Theorem 1.

In order to prove Theorem 2 we have to inject uniform bounds into the above argument. Let $\mathcal{F}$ denote both the admissible family of $L$-functions 
and the induced admissible family of data $(\widetilde{\boldsymbol{\lambda}}, \widetilde{\boldsymbol{\mu}})$, and let $F \in \mathcal{F}$. We follow the argument above with $K$ as in (2.4) and choosing $R$ and $L$ as in (3.8), but here $d$ denotes an upper bound for $d_{F}$ as $F \in \mathcal{F}$. We first remark that, in view of (1.3), the bound for $F(s, \alpha)$ is trivially true for $1+1 / 2 d_{F} \leq \sigma \leq 2$ since $1 \leq d_{F} \ll 1$, and hence we consider (3.2) in the range $-\Delta \leq \sigma<1+1 / 2 d_{F}$.

The first two terms on the RHS of (3.2) are dealt with by means of (1.4). By Stirling's formula, from (3.5) we deduce that for $k \leq m_{F}$ and $-\Delta \leq \sigma<$ $1+1 / 2 d_{F}$ with $|s-1| \geq 1 / 4$,

$$
\beta_{k}(s) \ll(|t|+2)^{c \Delta}|\log 2 \pi \alpha|^{m_{F}} \alpha^{(\sigma-1) d} .
$$

Moreover, by (3.3), (1.4) and Cauchy's formula for $k \leq m_{F}$ we have

$$
\alpha_{k} \ll q_{F}^{c},
$$

and hence by (3.4) for $-\Delta \leq \sigma<1+1 / 2 d_{F}$ with $|s-1| \geq 1 / 4$ we get

$$
R^{(1)}(s, \alpha) \ll q_{F}^{c}(|t|+2)^{c \Delta}|\log 2 \pi \alpha|^{m_{F}} \alpha^{(\sigma-1) d} .
$$

Further, by (1.4) and (3.7) for $n_{\alpha} \gg 1$ and $-\Delta \leq \sigma<1+1 / 2 d_{F}$ with $|s-1| \geq 1 / 4$ we have

$$
R^{(2)}(s, \alpha) \ll q_{F}^{c-\sigma}(|t|+2)^{c \Delta} \alpha^{c} .
$$

Therefore, since $R^{(1)}(s, \alpha)+R^{(2)}(s, \alpha)$ is holomorphic for $\sigma<1+1 / d_{F}$, from (3.10), (3.11) and the maximum modulus principle we have

$$
R^{(1)}(s, \alpha)+R^{(2)}(s, \alpha) \ll q_{F}^{c-\sigma}(|t|+2)^{c \Delta} \alpha^{c}
$$

uniformly for $F \in \mathcal{F},-\Delta \leq \sigma<1+1 / 2 d_{F}$ and $n_{\alpha} \gg 1$, with some constant $c=c(\mathcal{F})$.

We split the sum over $n$ on the RHS of (3.2) as in (3.9) and apply Theorem 2.1. Recalling that $Q^{2} \asymp q_{F}$, for $\left|s-s_{k}\right| \geq 1 / 4 d_{F}, k=0,1, \ldots$, the contribution of the first term in (3.9) to (3.2) is

$$
\ll q_{F}^{1 / 2-\sigma}\left|a\left(n_{\alpha}\right)\right| n_{\alpha}^{\sigma-1}(|t|+2)^{c \Delta} \ll q_{F}^{c} \alpha^{\sigma+c}(|t|+2)^{c \Delta}
$$

uniformly for $F \in \mathcal{F}$ and $-\Delta \leq \sigma<1+1 / 2 d_{F}$. The contribution of the second term in (3.9) to (3.2) is

$$
\begin{aligned}
& \ll q_{F}^{1 / 2-\sigma} \sum_{n \neq n_{\alpha}}|a(n)| n^{\sigma-1}(|t|+2)^{c \Delta}\left(\frac{n_{\alpha}}{n}\right)^{(K+1 / 2) / d}\left(\log \frac{n}{n_{\alpha}}\right)^{c \Delta / d} \\
& \ll q_{F}^{c-\sigma}(|t|+2)^{c \Delta} \delta(\alpha)^{c \Delta}
\end{aligned}
$$

uniformly for $F \in \mathcal{F},-\Delta \leq \sigma<1+1 / 2 d_{F}$ and $n_{\alpha} \gg 1$, and hence the required bound for $F(s, \alpha)$ follows from (3.2) and (3.12)-(3.14). 
Finally, by Theorem 1, Theorem $2.1,(3.2)$ and (3.9), for $k=0,1, \ldots$ we have

$$
\operatorname{res}_{s=s_{k}} F(s, \alpha)=\omega Q^{1-2 s_{k}} n_{\alpha}^{s_{k}-1} \overline{a\left(n_{\alpha}\right)} \frac{1}{d} \operatorname{res}_{s=s(k)} H_{K}\left(-\frac{i}{\widetilde{\beta}}, s ; \tilde{\boldsymbol{\lambda}}, \tilde{\boldsymbol{\mu}}\right)
$$

and, by Cauchy's integral formula,

$$
\operatorname{res}_{s=s(k)} H_{K}(-i / \widetilde{\beta}, s ; \widetilde{\boldsymbol{\lambda}}, \widetilde{\boldsymbol{\mu}}) \ll 1
$$

uniformly for $F \in \mathcal{F}$, where the implicit constant may also depend on $k$. The required expression for $\operatorname{res}_{s=s_{k}} F(s, \alpha)$ follows now from the definitions of $\omega_{F}^{*}$ and $q_{F}$.

4. Proof of the corollaries. The proof of Corollary 1 is standard, so we give only a sketch. Let $\phi(u)$ be a smooth function on $(0, \infty)$ with compact support and let

$$
\widetilde{\phi}(s)=\int_{0}^{\infty} \phi(u) u^{s-1} d u
$$

be its Mellin transform. It is well known that $\widetilde{\phi}(s)$ is an entire function satisfying

$$
\widetilde{\phi}(s) \ll(|t|+2)^{-C}, \quad a \leq \sigma \leq b,
$$

for every $C>0$. Starting from

$$
S_{F}(x)=\frac{1}{2 \pi i} \int F(s, \alpha) \widetilde{\phi}(s) x^{s} d s,
$$

we shift the line of integration to $\sigma=-\Delta$, where $\Delta>0$ is arbitrarily large and the line $\sigma=-\Delta$ has distance $\geq 1 / 4 d_{F}$ from the poles of $F(s, \alpha)$. Writing $r_{k}=\operatorname{res}_{s=s_{k}} F(s, \alpha)$, from Theorem 2 we get

$$
S_{F}(x)=\sum_{\Re s_{k} \geq-\Delta} r_{k} \widetilde{\phi}\left(s_{k}\right) x^{s_{k}}+O\left(q_{F}^{c+\Delta} \delta(\alpha)^{c \Delta} x^{-\Delta} \int_{-\infty}^{\infty}(|t|+2)^{c \Delta-C} d t\right),
$$

and Corollary 1 follows at once since $\Delta$ and $C$ are arbitrarily large.

In order to prove Corollary 2 we need a lemma concerning the function

$$
\zeta_{d}(s, \alpha)=\sum_{n=1}^{\infty} \frac{e\left(-n^{1 / d} \alpha\right)}{n^{s}}, \quad \sigma>1 .
$$

Lemma 4.1. For $d>1$ and $\alpha>0$ the function $\zeta_{d}(s, \alpha)$ is entire.

Proof. We first observe that the function

$$
Z_{d}(s, \alpha)=\pi^{s-1 / 2} \sum_{k=0}^{\infty} \frac{1}{k !} \frac{\Gamma\left(\frac{1}{2}\left(1-s+\frac{k}{d}\right)\right)}{\Gamma\left(\frac{1}{2}\left(s-\frac{k}{d}\right)\right)} \zeta(1-s+k / d)(2 \pi i \alpha)^{k}
$$


is entire. In fact, for each $k \geq 0$ the pole of $\zeta(1-s+k / d)$ is canceled by a zero of $\Gamma\left(\frac{1}{2}\left(s-\frac{k}{d}\right)\right)^{-1}$, and by Stirling's formula the series is rapidly convergent over $\mathbb{C}$ since $d>1$. Moreover, by Cauchy's theorem and the functional equation of $\zeta(s)$ (see also the beginning of the proof of Theorem 1 and Section 7 of [7]), for $\sigma>1$ we get

$$
\begin{aligned}
Z_{d}(s, \alpha)= & \lim _{N \rightarrow \infty} \frac{1}{2 \pi i} \int_{(2)} \pi^{s-1 / 2} \frac{\Gamma\left(\frac{1}{2}\left(1-s-\frac{w}{d}\right)\right)}{\Gamma\left(\frac{1}{2}\left(s+\frac{w}{d}\right)\right)} \\
& \times \Gamma(w) \zeta\left(1-s-\frac{w}{d}\right)\left(\frac{1}{N}+2 \pi i \alpha\right)^{-w} d w \\
= & \lim _{N \rightarrow \infty} \frac{1}{2 \pi i} \int_{(2)} \zeta\left(s+\frac{w}{d}\right) \Gamma(w)\left(\frac{1}{N}+2 \pi i \alpha\right)^{-w} d w \\
= & \zeta_{d}(s, \alpha),
\end{aligned}
$$

and the lemma follows.

We remark that Lemma 4.1 is due to Hardy [5] (see also n.3 of the Miscellaneous Examples at the end of Chapter IX of Titchmarsh [12]), although Hardy's proof is quite different from ours. We include the proof of Lemma 4.1 since it is a simplified version of our main idea in the proof of Theorem 1. In fact, in this case we have nice convergence properties of the hypergeometric function involved (corresponding to the case with "main parameter" $\mu<0$ in the sense of Braaksma [2]). We also remark that the method in the proof of Lemma 4.1 is general, in the sense that it provides the analytic continuation to $\mathbb{C}$ of the twist

$$
F_{d}(s, \alpha)=\sum_{n=1}^{\infty} \frac{a(n)}{n^{s}} e\left(-n^{1 / d} \alpha\right), \quad \sigma>1
$$

for every $F \in \mathcal{S}^{\sharp}$ and $d>d_{F}$. Indeed, the series

$$
\omega Q^{1-2 s} \sum_{k=0}^{\infty} \frac{1}{k !}\left(\prod_{j=1}^{r} \frac{\Gamma\left(\lambda_{j}(1-s+k / d)+\bar{\mu}_{j}\right)}{\Gamma\left(\lambda_{j}(s-k / d)+\mu_{j}\right)}\right) \bar{F}(1-s+k / d)(2 \pi i \alpha)^{k},
$$

which is the analog of (4.1), is rapidly convergent over $\mathbb{C}$ for $d>d_{F}$ and equals $F_{d}(s, \alpha)$ for $\sigma>1$.

Now we are ready for the proof of Corollary 2. We first remark that we may assume

$$
P(\log x)=\frac{1}{x} \operatorname{res}_{s=1} F(s) \frac{x^{s}}{s},
$$

otherwise the result is trivial since $x P(\log x)$ is the "wrong" main term. Moreover, since the functions in $\mathcal{S}_{1}^{\sharp}$ are suitable linear combinations of shifted Dirichlet $L$-functions over Dirichlet polynomials (see Theorem 2 of [7]), 
Corollary 2 holds true for $\mathcal{S}_{1}^{\sharp}$, and hence we may restrict our attention to functions $F \in \mathcal{S}_{d}^{\sharp}$ with $d>1$. As in (3.3) we write

$$
F(s)=\sum_{k=1}^{m_{F}} \frac{\alpha_{k}}{(s-1)^{k}}+f_{1}(s)
$$

with $f_{1}(s)$ entire (the sum is empty if $m_{F}=0$ ). Moreover, we write

$$
c(n)=a(n)-\sum_{k=0}^{m_{F}-1} \frac{\alpha_{k+1}}{k !} \log ^{k} n, \quad H(s)=\sum_{n=1}^{\infty} \frac{c(n)}{n^{s}} .
$$

Clearly

$$
H(s)=F(s)-\sum_{k=0}^{m_{F}-1} \frac{(-1)^{k}}{k !} \alpha_{k+1} \zeta^{(k)}(s),
$$

and hence $H(s)$ is an entire function. Suppose now that Corollary 2 is false, i.e.

$$
A_{F}(x)-x P(\log x)=o\left(x^{(d-1) / 2 d}\right) .
$$

Therefore, by well known asymptotic formulae for the mean value of $\log ^{k} n$ and since $d>1$, by computing $P(\log x)$ in (4.2) we get

$$
\sum_{n \leq x} c(n)=o\left(x^{(d-1) / 2 d}\right) .
$$

Hence, for $s$ in a compact set, by partial summation we have

$$
\sum_{n>y} c(n) n^{-s}=o\left(y^{(d-1) / 2 d-\sigma}\right), \quad \sigma>\frac{d-1}{2 d} .
$$

For $\bar{\alpha}>0$ with $a\left(n_{\bar{\alpha}}\right) \neq 0$ we consider the function

$$
H(s, \bar{\alpha})=\sum_{n=1}^{\infty} \frac{c(n)}{n^{s}} e\left(-n^{1 / d} \bar{\alpha}\right)=F(s, \bar{\alpha})-\sum_{k=0}^{m_{F}-1} \frac{(-1)^{k}}{k !} \alpha_{k+1} \zeta_{d}^{(k)}(s, \bar{\alpha}) .
$$

By partial summation, for $\sigma>1$ we get

$$
H(s, \bar{\alpha})-e(-\bar{\alpha}) H(s)=-\frac{2 \pi i \bar{\alpha}}{d} \int_{1}^{\infty}\left(\sum_{n>y} \frac{c(n)}{n^{s}}\right) y^{1 / d-1} e\left(-y^{1 / d} \bar{\alpha}\right) d y,
$$

and in view of (4.3) such a formula holds for $\sigma>(d+1) / 2 d$ since the integral is convergent there. Moreover, for $\sigma>(d+1) / 2 d$ we have

$$
\begin{array}{r}
H(s, \bar{\alpha})-e(-\bar{\alpha}) H(s)=o\left(\int_{1}^{\infty} y^{-(d-1) / 2 d-\sigma} d y\right)=o\left(\frac{1}{\sigma-(d+1) / 2 d}\right), \\
\sigma \rightarrow\left(\frac{d+1}{2 d}\right)^{+} .
\end{array}
$$


But, in view of Theorem 1 and Lemma 4.1, $H(s, \bar{\alpha})$ has a simple pole at $s_{0}=(d+1) / 2 d-i \theta_{F} / d$, while $H(s)$ is entire. Therefore, for $s=\sigma-i \theta_{F} / d$ we have

$$
H(s, \bar{\alpha})-e(-\bar{\alpha}) H(s) \gg \frac{1}{\sigma-(d+1) / 2 d}, \quad \sigma \rightarrow\left(\frac{d+1}{2 d}\right)^{+},
$$

a contradiction in view of (4.4), and Corollary 2 follows.

In the proof of Corollary 3 we may assume that $F(s)$ is entire, otherwise the result is trivial. Since the convergence of the series

$$
\sum_{n=1}^{\infty} a(n)
$$

is excluded by Corollary 2 with the choice $P(x)=0$ identically, we have

$$
\sigma_{c}(F)=\limsup _{x \rightarrow \infty} \frac{\log \left|A_{F}(x)\right|}{\log x}
$$

(see Section 9.14 of Titchmarsh [12]). Hence Corollary 3 follows at once from Corollary 2, again with the choice $P(x)=0$ identically. The generalization of Corollary 3 follows by the same argument applied to the function

$$
G(s)=F(s)-\sum_{j=0}^{k}(-1)^{j} c_{j} \zeta^{(j)}(s),
$$

where $Q(x)=c_{0}+c_{1} x+\cdots+c_{k} x^{k}$.

Finally, the proof of the first part of Corollary 4 uses the generalization of Corollary 3. In fact, the abscissa of convergence $\sigma_{c}(F-\zeta)$ of the Dirichlet series

$$
F(s)-\zeta(s)=\sum_{n=1}^{\infty} \frac{a(n)-1}{n^{s}}
$$

satisfies $\sigma_{c}(F-\zeta) \geq\left(d_{F}-1\right) / 2 d_{F}$. Since $\mathcal{S}_{d}=\emptyset$ for $1<d<5 / 3$ (see [9]) if $d_{F}>1$ then $d_{F} \geq 5 / 3$, and hence $\sigma_{c}(F-\zeta) \geq 1 / 5$. This contradicts our assumption that (4.5) converges for $\sigma<1 / 5-\delta$, and hence $d_{F}=1$. Moreover, the convergence of (4.5) for $\sigma<1 / 5-\delta$ implies that $F(s)$ has a pole at $s=1$, and the result follows since $\zeta(s)$ is the only function in $\mathcal{S}_{1}$ with a pole at $s=1$ (see Theorem 3 of [7]). The second part of Corollary 4 is proved in the same way, with the difference that in this case $F(s)$ is entire, and hence from Theorem 3 of [7] we deduce that $F(s)$ is a shift of a Dirichlet $L$-function formed with a primitive character. 


\section{References}

[1] S. Bochner, On Riemann's functional equation with multiple gamma factors, Ann. of Math. 67 (1958), 29-41.

[2] B. L. J. Braaksma, Asymptotic expansions and analytic continuations for a class of Barnes-integrals, Compositio Math. 15 (1964), 239-341.

[3] K. Chandrasekharan and R. Narasimhan, Functional equations with multiple gamma factors and the average order of arithmetical functions, Ann. of Math. 76 (1962), 93-136.

[4] J. B. Conrey and A. Ghosh, On the Selberg class of Dirichlet series: small degrees, Duke Math. J. 72 (1993), 673-693.

[5] G. H. Hardy, Theorems connected with Maclaurin's test for the convergence of series, Proc. London Math. Soc. (2) 9 (1911), 126-144.

[6] H. Iwaniec, W. Luo and P. Sarnak, Low lying zeros of families of L-functions, Inst. Hautes Études Sci. Publ. Math. 91 (2000), 55-131.

[7] J. Kaczorowski and A. Perelli, On the structure of the Selberg class, I: $0 \leq d \leq 1$, Acta Math. 182 (1999), 207-241.

[8] —, 一, The Selberg class: a survey, in: Number Theory in Progress, Proc. Conf. in Honor of A. Schinzel, K. Győry et al. (eds.), de Gruyter, 1999, 953-992.

[9] - - - On the structure of the Selberg class, $V: 1<d<5 / 3$, Invent. Math. 150 (2002), 485-516.

[10] G. Molteni, A note on a result of Bochner and Conrey-Ghosh about the Selberg class, Arch. Math. (Basel) 72 (1999), 219-222.

[11] H.-E. Richert, Über Dirichletreihen mit Funktionalgleichung, Publ. Inst. Math. Acad. Serbe Sci. 11 (1957), 73-124.

[12] E. C. Titchmarsh, The Theory of Functions, 2nd ed., Oxford Univ. Press, 1939.

Faculty of Mathematics and Computer Science

A. Mickiewicz University

Umultowska 87

61-614 Poznań, Poland

E-mail: kjerzy@amu.edu.pl
Dipartimento di Matematica

Via Dodecaneso 35

16146 Genova, Italy

E-mail: perelli@dima.unige.it 